Sunday Symposia, and the entirety of the Annual Meeting simultaneous sessions. Register for a Saturday course and/or a Sunday Symposium to have access to all courses/symposia taking place that same day. Please note registration for the Saturday courses and/or Sunday Symposium is separate from the Annual Meeting registration rate.

View the preliminary program, register and reserve housing at aats.org/annualmeeting

\section{AATS Surgical Patient Safety Course 2018}

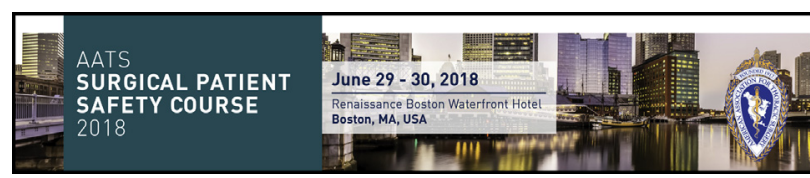

June 29-30, 2018

Renaissance Boston Waterfront Hotel

Boston, MA

Co-sponsored by:

Society of Cardiovascular Anesthesiologists

In collaboration with:

American Society of Extracorporeal Technology

Association of periOperative Registered Nurses

Association of Physician Assistants In Cardiovascular Surgery

At the AATS Surgical Patient Safety Course, you and your surgical team can gain essential knowledge and skills while contributing to improving an area of constant concern for all members of the surgical team.

Take advantage of this opportunity not only to learn with your team but also to share your research and scholarly activity in topical areas of patient safety in cardiothoracic surgery by submitting an abstract today. If accepted, you will be a part of an exciting forum of significant and innovative research, and have the opportunity to identify collaborators and receive feedback on your work.

Program topics include:

- Surgical technique

- Nontechnical skills

- Quality improvement

- Education

- Simulation

- Outcomes

- Data science

- Machine learning

- Technology development

- Human factors

- Health services research
Abstract Submission is now open.

Submission Deadline: Monday, May 11, 2018, at 11:59 PM Eastern Time

Abstract submission guidelines and additional information can be found at www.aats.org/patientsafety

\section{AATS Clinical Trials Methods Course}

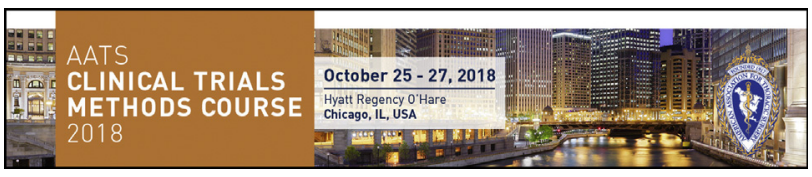

Registration \& Housing Now Available

October 25-27, 2018

Hyatt Regency O'Hare

Chicago, IL

Program Directors

David H. Harpole, Jr

Marco A. Zenati

\section{Program Overview}

This intensive and interactive training program is aimed at cardiothoracic surgeons across subspecialties, especially those who are planning to apply for clinical trial funding. The course will help attendees to better understand the complex nature of clinical trial proposal preparation and submission. It will also delineate a process for achieving streamlined protocol development. Interactive features will include hands-on focus groups and mock study sessions.

More information: www.aats.org/clinicaltrials

\section{AATS Focus on Thoracic Surgery: Lung and} Esophageal Cancer

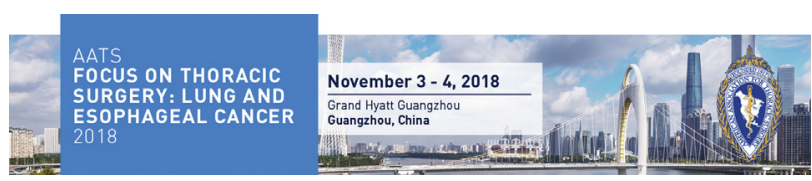

\section{SAVE THE DATE}

November 3-4, 2018

Grand Hyatt Guangzhou

Guangzhou, China

Program Directors

Jianxing $\mathrm{He}$

G. Alexander Patterson

David J. Sugarbaker

\section{Program Overview}

Lung cancer and esophageal cancer remain a global concern for patients and thoracic surgeons as two of the 